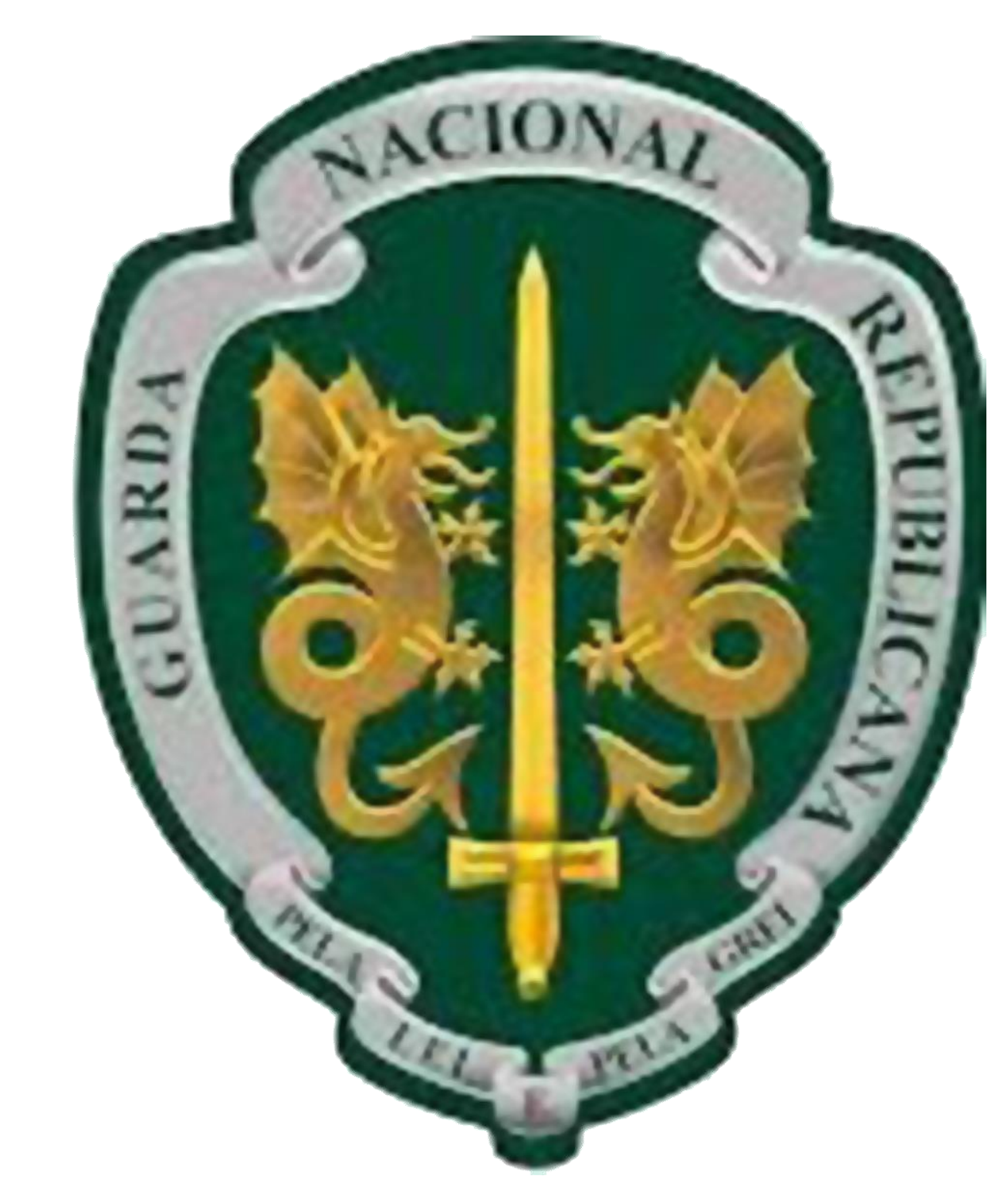

\title{
A multiple session mesotherapy protocol for the management of hip osteoarthritis related pain in police working dogs
}

\author{
Alves J. ${ }^{1,2, *}$; Santos A. ${ }^{1}$; Jorge P. ${ }^{1}$; Lafuente P. ${ }^{3}$
}

'Guarda Nacional Republicana; ${ }^{2}$ ICAAM - Instituto de Ciências Agrárias e Ambientais Mediterrâneas, Universidade de Évora; ${ }^{3}$ Department of Animal Medicine and Surgery - Universidad CEU Cardenal Herrera; *alves.jca@gnr.pt

\section{'OJ BJECCIIVIES:}

To describe the use and effectiveness of mesotherapy in working dogs with hip osteoarthritis (OA).

\section{MATERIALS AND METHODS:}

Thirty dogs were assigned to a control group (CG, $\mathrm{n}=10$ ) or a treatment group (MG, $\mathrm{n}=20$ ). CG received a 70 day course of meloxicam, and MG was treated with a combination of lidocaine, piroxicam and thiocolchicoside;

The solution was injected in multiple intradermal points over the hip area, laterally and medially. Mesotherapy sessions were conducted once a week for four sessions, and every other week for three sessions.

Response to treatment, measured by the Canine Brief Pain Inventory (CBPI, divided in Pain Interference Score - PIS, and Pain Severity Score - PSS), Liverpool OA in dogs (LOAD) and the Hudson Visual Analogue Scale (HVAS), was evaluated before treatment, $+15,+30,+60$ and +90 days after initial treatment.

Results were analyzed with the Wilcoxon signed ranks test, and Kaplan-Meier estimators were conducted and compared with the Breslow test, with $p<0.05$.

\section{RESULTS:}

No significant differences were observed between groups at the initial evaluation. Better results were observed in MG at $+15 d(p=0.01$ for HVAS, and $p<0.01$ for PSS and PIS), $+30 d$ ( $p=0.04$ for HVAS, and $p=0.02$ for PIS, $p=0.03$ for PSS and LOAD), $+60 d$ ( $p<0.05$ for HVAS and LOAD, $p<0.01$ for PIS and PSS) and $+90 d$ ( $p=0.02$ for PIS and PSS).

Kaplan-Meier estimators showed significantly better results in the MG group. No side-effects were recorded.
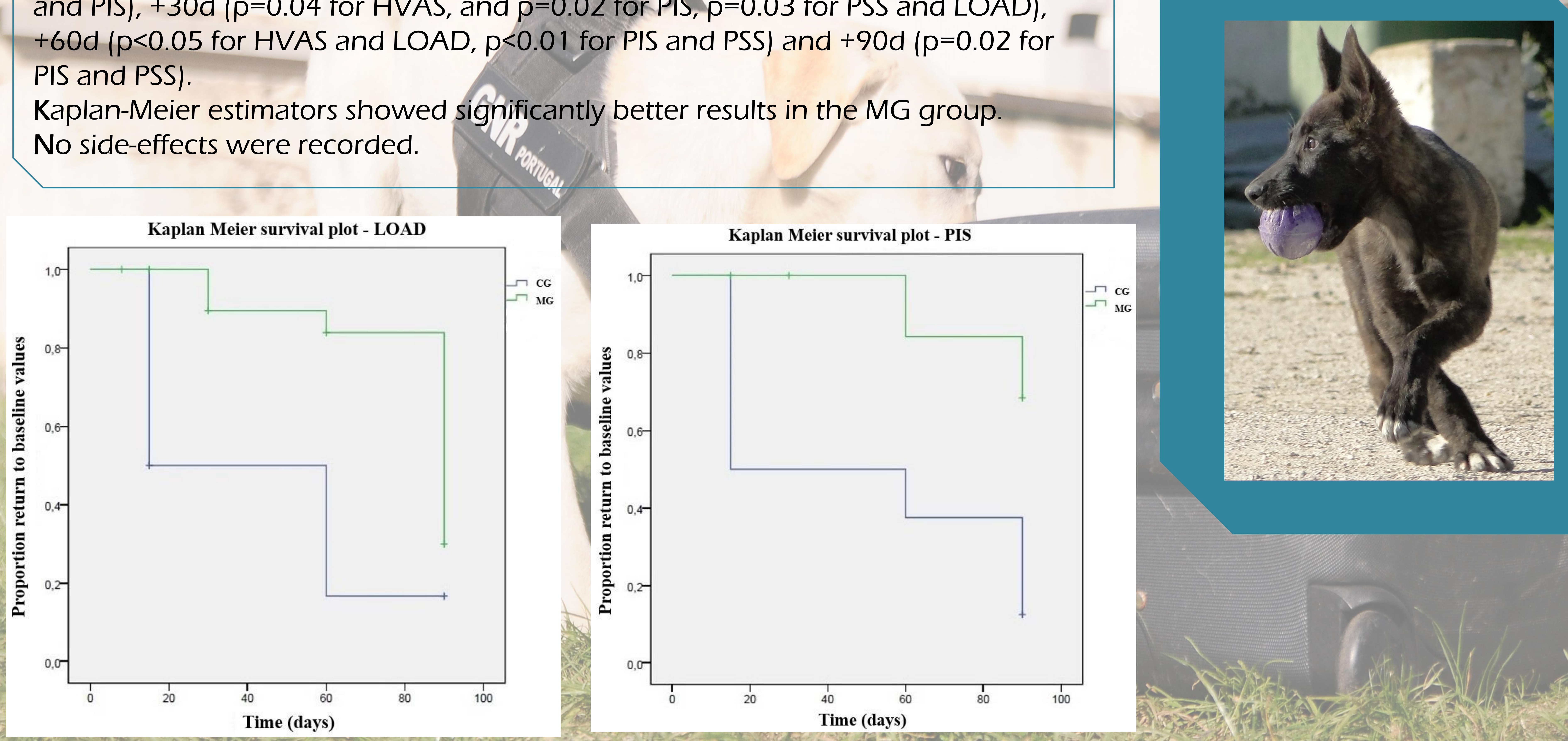